\title{
Inhibiting a spinal cord signaling pathway protects against ischemia injury in rats
}

\author{
Jia Huo, MD, ${ }^{\text {a }}$ Rui Ma, MD, ${ }^{\mathrm{b}}$ Xin Chai, MD, ${ }^{\mathrm{a}}$ Hong-Jin Liang, MD, ${ }^{\mathrm{a}}$ Peng Jiang, MD, ${ }^{\mathrm{a}}$ Xiao-ling Zhu, PhD, ${ }^{\mathrm{a}}$ \\ $\mathrm{Xin}$ Chen, $\mathrm{PhD},{ }^{\mathrm{c}}$ and Bin-Xiao $\mathrm{Su}, \mathrm{PhD}^{\mathrm{a}}$
}

\section{ABSTRACT}

Objective: The aim of the study was to examine whether the cannabinoid agonist WIN55212-2 could attenuate ischemic spinal cord injury (SCI) in rats through inhibition of GAPDH/Siah1 signaling.

Methods: Male Sprague-Dawley rats were distributed randomly into 5 groups: (1) sham group that received no aortic occlusion and injected intraperitoneally (i.p.) with vehicle control after reperfusion; (2) control group that received a 12-minute aortic occlusion and injected i.p. with vehicle control after reperfusion; (3) WIN55212-2 group (WIN) that received the aortic occlusion and injected i.p. with $1 \mathrm{mg} / \mathrm{kg}$ of WIN55212-2 after reperfusion; and (4) WIN55212-2 plus AM251 group and (5) WIN55212-2 plus AM630 group that received the same surgical operation as the WIN group, except that $1 \mathrm{mg} / \mathrm{kg}$ of AM251 or AM630 was injected i.p. 30 min before each dose of WIN55212-2 injection, respectively. Neurologic function was assessed 48 hours after reperfusion. Histopathologic examination was performed to determine the number of normal neurons in anterior spinal cord. Protein expression of active caspase-3, total caspase-3, glyceraldehyde 3-phosphate dehydrogenase (GAPDH), inducible nitric oxide synthase (iNOS), nuclear factor kappa light chain enhancer of activated B cells $(\mathrm{NF}-\kappa \mathrm{B})$, Siah1, tumor necrosis factor $\alpha$, and interleukin $1 \beta$ were determined with Western blot and enzyme-linked immunosorbent assay; coimmunoprecipitation assays were also used to determine GAPDH/Siah1 complexing. Finally, terminal deoxynucleotidyl transferase dUTP nick end labeling staining was used to determine neuronal apoptosis in the lumbar spinal cord.

Results: The nuclear translocation of GAPDH and Siah1 in the spinal cord was initiated after ischemic spinal cord injury (SCI) along with the increased formation of GAPDH/Siah1 complexes. However, the activation of GAPDH/Siah1 was blocked by WIN. In addition, the treatment of WIN55212-2 promoted neuronal survival in the spinal cord, reduced apoptosis and inflammation, and improved neurologic scores. Furthermore, these beneficial effects of WIN55212-2 were abolished by the combined treatment of the CB2 antagonist AM630, but not the CB1 antagonist AM251.

Conclusions: Our findings reveal GAPDH/Siah1 signaling cascades as a novel therapeutic target for ischemic SCI and identify WIN55212-2 with the potential to treat ischemic SCI by targeting this pathway. (J Thorac Cardiovasc Surg 2019;157:494-503)

From the ${ }^{\mathrm{a}}$ Department of Anesthesiology, Xijing Hospital, Fourth Military Medical University, Xi'an, China; 'Department of Anesthesiology, Xi' an Children's Hospital, Xi' an, China; and 'Department of Anesthesiology, Shaanxi Provincial People's Hospital, Xi'an, China.

Supported by a grant from the National Natural Science Foundation of China (grant number 81100901).

Drs Huo and Ma contributed equally to this work and are considered co-first authors. Received for publication March 18, 2018; revisions received July 12, 2018; accepted for publication July 13, 2018; available ahead of print Sept 6, 2018.

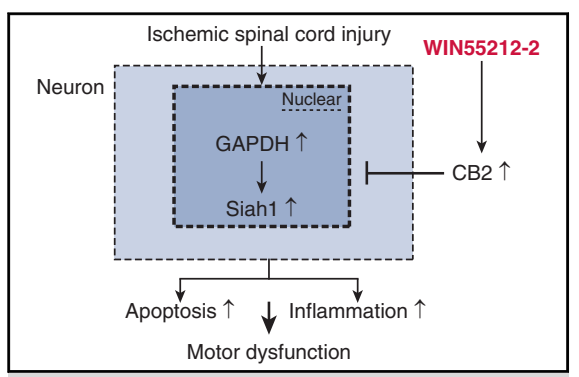

WIN55212-2 attenuates ischemic SCI by inhibiting $\mathrm{GAPDH} / \mathrm{Siah} 1$ via the $\mathrm{CB} 2$ receptor.

\section{Central Message}

WIN55212-2 protects against ischemic spinal cord injury in rats. The neuroprotective effect of WIN55212-2 depends on cannabinoid CB2 receptor-mediated inhibition of GAPDH/ Siah1 signaling.

\section{Perspective}

Our results reveal GAPDH/Siah1 cascades as a novel therapeutic target for ischemic SCI and identify WIN55212-2 or other synthetic cannabinoid agonist with the potential to manage ischemic SCI or other devastating ischemic conditions.

See Editorial page 504.

See Editorial Commentary page 506.

\footnotetext{
Address for reprints: Xin Chen, PhD, Department of Anesthesiology, Shaanxi Provincial People's Hospital, Xi'an 710068, China (E-mail: 86887243@qq.com); or Bin-Xiao Su, PhD, Department of Anesthesiology, Xijing Hospital, Fourth Military Medical University, Xi' an 710032, China (E-mail: Su_luccy@126.com). $0022-5223 / \$ 36.00$

Copyright (C) 2018 by The American Association for Thoracic Surgery https://doi.org/10.1016/j.jtcvs.2018.07.045
} 


$\begin{array}{ll}\text { Abbreviations and Acronyms } \\ \text { CNS } & =\text { central nervous system } \\ \text { GAPDH } & =\text { glyceraldehyde 3-phosphate } \\ & \text { dehydrogenase } \\ \text { IL-1 } \beta & =\text { interleukin } 1 \text { beta } \\ \text { iNOS } & \text { nitric oxide synthase } \\ \text { I } \kappa \text { B- } \alpha & =\text { nuclear factor of kappa light } \\ & \text { polypeptide gene enhancer in B-cells } \\ & \text { inhibitor, alpha } \\ \text { i.p. } & =\text { intraperitoneal } \\ \text { IP } & =\text { immunoprecipitation } \\ \text { IR } & =\text { ischemia-reperfusion } \\ \text { MDI } & =\text { motor deficit index } \\ \text { NF- } \kappa \text { B } & =\text { nuclear factor kappa-light-chain- } \\ & \text { enhancer of activated B cells } \\ \text { NO } & =\text { nitric oxide } \\ \text { SCI } & =\text { spinal cord injury group } \\ \text { SDS-PAGE }= & \text { sodium dodecyl sulfate- } \\ & \text { polyacrylamide gel electrophoresis } \\ \text { Siah1 } & =\text { E3 ubiquitin-protein ligase SIAH1 } \\ \text { TNF- } \alpha & =\text { tumor necrosis factor-alpha } \\ \text { TUNEL } & =\text { Terminal deoxynucleotidyl } \\ & \text { transferase dUTP nick end labeling }\end{array}$

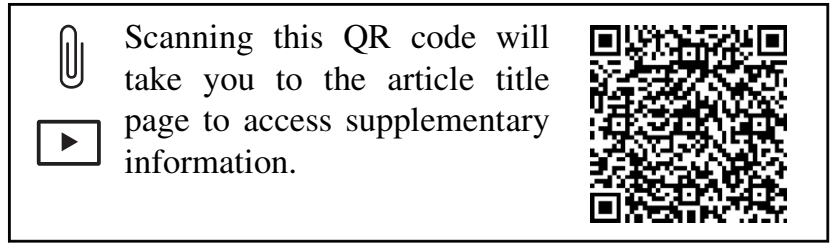

Ischemia-reperfusion (IR) injury is the main cause of paraplegia after operations on thoracoabdominal aortic aneurysms. Although many new techniques have been developed to reduce this serious complication, prevention of ischemic spinal cord injury is still a major concern for thoracic aorta surgery.

The mechanisms of IR injury have also been widely investigated. Among them, the cannabinoid system with its receptors (CB1 and CB2), have been demonstrated to provide neuroprotection against cerebral ${ }^{1}$ and spinal $\operatorname{cord}^{2,3}$ IR injuries. WIN55212-2 (WIN) is an agonist for CB1 and CB2 receptors, and it has demonstrated inhibitory effects on neutrophil infiltration into the central nervous system (CNS) during neurologic diseases, such as multiple sclerosis. ${ }^{4,5}$ Furthermore, WIN55212-2 also reduces apoptosis and inflammation, with evidence of promoting neural repair in neonatal animals experiencing hypoxia-ischemia. ${ }^{6}$ Finally, WIN55212-2 can also relieve neuropathic pain after compression-induced spinal cord injury $(\mathrm{SCI})^{7}$ or peripheral nerve injury. ${ }^{8}$ Mounting evidence indicates that WIN55212-
2 possessed significant neurotrophic and neuroprotective properties; therefore, it is possible that WIN55212-2 treatment could also attenuate spinal cord IR injury.

Glyceraldehyde-3-phosphate dehydrogenase (GAPDH) was originally thought as merely a housekeeping enzyme constitutively present in the cytosol. However, emerging studies have shown that the nuclear translocation of GAPDH could be induced by nitric oxide (NO) to complex with Siah1 (an E3-ubiquitin-ligase), causing the activation of apoptotic signaling cascades. ${ }^{9,10}$ Previous studies have indicated that GAPDH/Siah1 signaling cascades were correlated with the progression of various diseases, including cerebral IR injury ${ }^{11}$ and acute lung injury. ${ }^{12}$ Importantly, our latest study has shown that GAPDH/ Siah1 signaling cascades are involved in the initiation of apoptosis and inflammation in the spinal cord after traumatic SCI. ${ }^{13}$ Interestingly, many studies have found that activation of the cannabinoid system can suppress NO synthesis, ${ }^{14-16}$ which can in turn regulate GAPDH/Siah1 signaling cascades. Thus, the aim of this study was to determine whether the cannabinoid WIN55212-2 would have a therapeutic effect on ischemic SCI. We hypothesized that WIN55212-2 treatment would attenuate ischemic SCI and reduce apoptosis and inflammation in the spinal cord through inhibition of GAPDH/Siah1 signaling.

\section{MATERIALS AND METHODS \\ Animals}

The study was performed in accordance with the Guidelines for Animal Experimentation of the Fourth Military Medical University (Xi'an, China), and the experimental protocols were approved by the Ethics Committee for Animal Experimentation of the Fourth Military Medical University. Male Sprague-Dawley rats weighing 300-350 g were purchased from the Experimental Animal Center of the Fourth Military Medical University. The rats were kept in a temperature- and humidity-controlled environment with free access to a standard diet and water.

\section{Surgical Procedure}

The IR injury of the spinal cord was induced as described in our previous study. ${ }^{3}$ The rats were anesthetized with $4 \%$ isoflurane in oxygen and then maintained with $1.5 \%$ to $2 \%$ isoflurane. The body temperature was kept at $37^{\circ} \mathrm{C}$ with a homeothermic blanket system. Distal arterial blood pressure was monitored with a tail artery catheter (Colin BP 508; Colin Corporation, Komaki City, Japan). With the animal under anesthesia, a 2-French Fogarty catheter (American V. Mueller, CV 1035; Baxter, Irvine, Calif) was inserted through the left femoral artery to the descending thoracic aorta for 10.8 to $11.4 \mathrm{~cm}$, allowing the catheter tip to reach the level of the left subclavian artery. The balloon was then inflated with $0.20 \mathrm{~mL}$ of air to form an occlusion, which was confirmed by an immediate and sustained drop in distal arterial blood pressure. After $12 \mathrm{~min}$ of ischemia, the balloon was deflated to recover the spinal cord blood flow. Finally, the arterial lines were removed, wounds were closed, and the animals were allowed to recover The surgical procedure is demonstrated in Video 1.

\section{Experimental Groups}

The rats were randomly assigned into 5 groups (Figure 1, A): (1) sham group (Sham) in which the rats only received the surgical procedure but without aortic occlusion, and were injected intraperitoneally (i.p.) with 


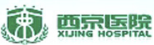

Surgical procedure of rat spinal cord ischemic injury

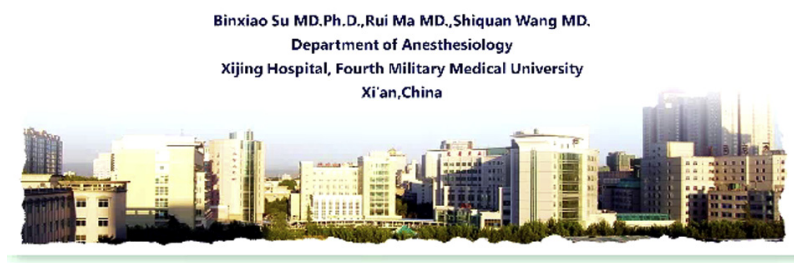

VIDEO 1. The rats were anesthetized with $4 \%$ isoflurane in oxygen and then maintained with $1.5 \%$ to $2 \%$ isoflurane. The body temperature was kept at $37^{\circ} \mathrm{C}$ with a homeothermic blanket system. Distal arterial blood pressure was monitored by a tail artery catheter. After administering anesthesia, a 2-French Fogarty catheter (American V. Mueller, CV 1035; Baxter, Irvine, Calif) was inserted through left femoral artery to the descending thoracic aorta at the distance of $10.8-11.4 \mathrm{~cm}$, allowing the catheter tip to reach the level of the left subclavian artery. Next, the balloon was inflated with $0.20 \mathrm{~mL}$ of air. The successful occlusion was confirmed by an immediate and sustained drop in distal arterial blood pressure. After 12 minutes of ischemia, the balloon was deflated to recover the spinal cord blood flow. Next, the arterial lines were removed, the wounds were closed, and the animals were allowed to recover. Video available at: https://www.jtcvs.org/ article/S0022-5223(18)32035-X/fulltext.

$0.5 \mathrm{~mL}$ of the vehicle solution ( $90 \%$ saline, $5 \%$ dimethyl sulfoxide, and $5 \%$ Tween 80 ) immediately, 8 hours, and 24 hours after reperfusion; (2) control group (Control) in which the rats received the 12-minute aortic occlusion and were injected i.p. with $0.5 \mathrm{~mL}$ of the vehicle solution immediately, 8 hours, and 24 hours after reperfusion; (3) WIN55212-2 group (WIN) in which the rats received the 12-minute aortic occlusion and were injected i.p. with $1 \mathrm{mg} / \mathrm{kg}$ of WIN55212-2 (catalog number W102; Sigma Chemical, St Louis, Mo) immediately, 8 hours, and 24 hours after reperfusion; (4) WIN55212-2 plus AM251 group (WIN+AM251) in which the operation was the same as that of WIN, except that a $1-\mathrm{mg} / \mathrm{kg}$ dose of AM251 (CB1 receptor antagonist; Tocris Bioscience, Bristol, United Kingdom) was injected i.p. 30 min before each WIN55212-2 injection; (5) WIN55212-2 plus AM630 group (WIN+AM630) in which the operation was the same as that for WIN, except that a $1-\mathrm{mg} / \mathrm{kg}$ dose of AM630 (CB2 receptor antagonist; Tocris Bioscience) was injected i.p. $30 \mathrm{~min}$ before each dose of WIN55212-2 injection. WIN55212-2, AM251, and AM630 were prepared in vehicle solution. The dose of WIN55212-2 was based on the study by Hama and Sagen, ${ }^{7}$ while the doses of AM251 and AM630 were based on our previous study. ${ }^{3}$

To optimize the best time for the molecular analysis, we evaluated GAPDH/Siah expression 24, 48, and 72 hours after reperfusion. These data showed that the nuclear expressions of GAPDH increased after reperfusion, peaked at 24 hours, and then began to decrease (Figure E1; $P<.01$; $\mathrm{n}=4$ per group). The cytoplasmic GAPDH expressions decreased after SCI, reaching a minimum at 24 hours (Figure $\mathrm{E} 1 ; P<.01 ; \mathrm{n}=4$ per group). These observations led to the selection of 24 hours after reperfusion as the point for additional experiments.

\section{Neurologic and Histopathologic Evaluations}

The neurologic evaluations were performed 48 hours after reperfusion by an investigator who was blinded to the experimental groups $(n=10$ per group). The motor function deficits in the hind limbs were assessed against a 14-point motor deficit score. The criteria are as follows: (a) walking or use of hind limbs: $0=$ normal, $1=$ toes flat under the body when walking, 2 = knuckle walking, $3=$ movement of the hind limbs but unable to walk, and $4=$ no movement, dragging hind limbs; and (b) placing or stepping reflex: $0=$ normal, $1=$ weak, and $2=$ not stepping. The motor deficit scores were obtained by adding the scores in scales (a) and (b), leading to a final motor deficit index (MDI). In addition, histopathologic evaluations were performed for the number of normal neurons in the spinal cord 48 hours after reperfusion. The rats were anesthetized with sodium pentobarbital $(60 \mathrm{mg} / \mathrm{kg})$. Transcardiac perfusions were then performed with $200 \mathrm{~mL}$ of saline solution followed by $250 \mathrm{~mL}$ of $4 \%$ paraformaldehyde. The spinal cords were dissected following fixation and were post-fixed in the same fixative overnight. Sections were taken from the L4 to L6 segments and stained with hematoxylin and eosin. The number of normal neurons in the anterior spinal cord of 3 sections selected randomly from the L4 to L6 segment was counted at a magnification of $200 \times$ based on the morphologic appearance and then averaged. The data are presented as the number of normal neurons per section.

\section{Western Blot}

The rats were first deeply anesthetized with sodium pentobarbital $(80 \mathrm{mg} /$ kg, i.p.) and then euthanized with $\mathrm{CO}_{2}$ asphyxiation ( $\mathrm{n}=4$ or 5 per group). The total protein from the spinal cord tissue of the lesion epicenter was extracted with homogenization in ice-cold radioimmunoprecipitation assay lysis buffer (Beyotime, Nantong, China) with a complete protease inhibitor cocktail (Roche Diagnostics, Indianapolis, Ind) and $1 \mathrm{mM}$ phenylmethylsulfonyl fluoride (PMSF). Nucleic and cytoplasmic proteins were extracted with the Nuclear and Cytoplasmic Extraction Reagents kit (Pierce Biotechnology, Rockford, Ill) following the manufacturer's instructions. Equal amounts of the protein sample were resolved on $12 \%$ sodium dodecyl sulfate-polyacrylamide gel electrophoresis (SDS-PAGE), transferred onto polyvinylidene difluoride membranes (Bio-Rad, Richmond, Calif), and detected with enhanced chemiluminescence Western blotting detection reagents (Thermo Scientific, Rockford, Ill) and quantified via densitometry. The following primary antibodies were used in the study: active (cleaved) caspase-3 antibody (Cell Signaling Technology, Beverly, Mass; 1:500), total caspase-3 antibody (Cell Signaling Technology; 1:1000), $\beta$-actin antibody (Santa Cruz Biotechnology, Santa Cruz, Calif; 1:1000), GAPDH antibody (Abcam, Cambridge, Mass, USA; 1:1000), histone $\mathrm{H}_{2} \mathrm{~B}$ antibody (Abcam; 1:1000), inducible nitric oxide synthase (iNOS) antibody (Abcam; 1:500), $\mathrm{I} \kappa \mathrm{B}-\alpha$ antibody (Cell Signaling Technology; 1:1000), NF- $\kappa \mathrm{B}$ antibody (Cell Signaling Technology; 1:500), and goat Siah1 antibody (Abcam; 1:1000). The $\beta$-actin and histone $\mathrm{H}_{2} \mathrm{~B}$ antibodies served as the loading controls of cytoplasmic and nucleic protein, respectively.

\section{Coimmunoprecipitation Assay}

Total protein extracts were prepared from spinal cord tissue of the lesion epicenter, followed by homogenization with immunoprecipitation buffer (Beyotime) supplemented with $1 \mathrm{mM}$ PMSF protease inhibitor for 1 hour. After centrifugation at 12,000 rpm for 10 minutes, $200 \mu \mathrm{g}$ of the protein extract was incubated with $5 \mu \mathrm{g}$ of goat anti-Siah1 polyclonal antibody (Abcam) overnight at $4{ }^{\circ} \mathrm{C}$, followed by precipitation with protein $\mathrm{A} /$ G-agarose (Millipore, Billerica, Mass) for 3 hours. After washing with the immunoprecipitation buffer and heating at $95^{\circ} \mathrm{C}$ for 10 minutes, the immunoprecipitates were subjected to SDS-PAGE and immunoblotting, followed by Densitometric quantification ( $\mathrm{n}=4$ per group).

\section{Enzyme-Linked Immunosorbent Assay}

The protein extraction procedure for the enzyme-linked immunosorbent assay (ELISA) was the same as that for Western blotting. The protein levels of tumor necrosis factor-alpha (TNF- $\alpha$ ) and interleukin (IL)- $1 \beta$ were determined with commercial ELISA kits (R\&D Systems, Minneapolis, Minn) according to the manufacturer's instructions. All samples were assayed in duplicate, and the final concentration was expressed as picograms per milligram of protein ( $\mathrm{n}=5$ per group). 


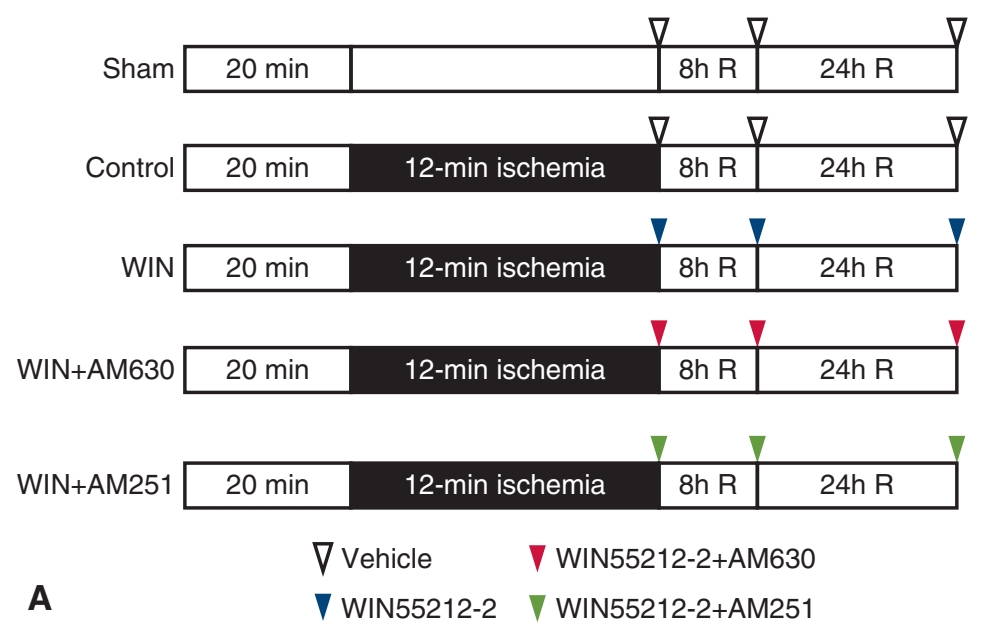

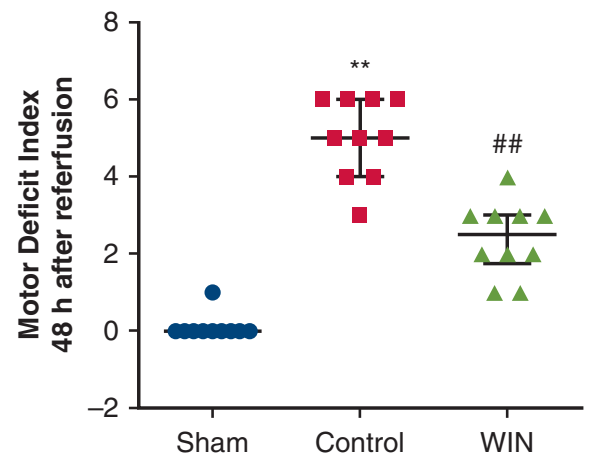

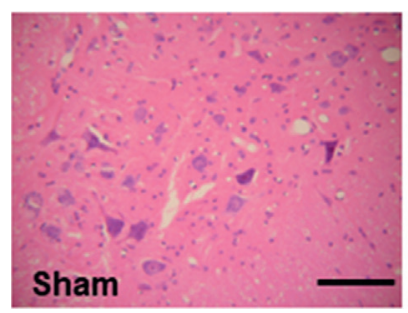

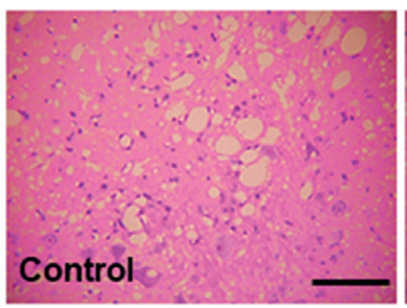

C

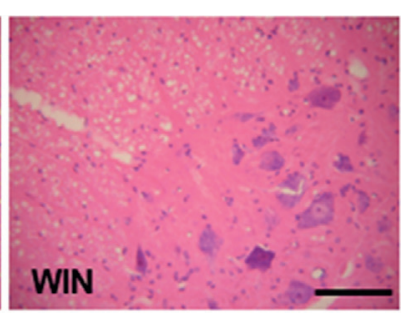

B

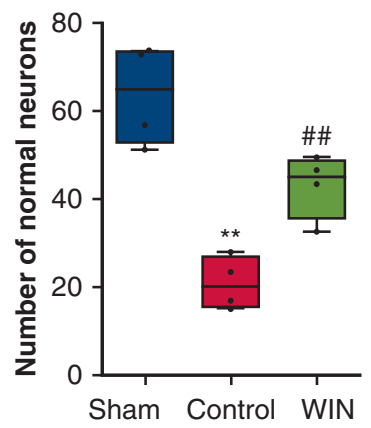

FIGURE 1. WIN55212-2 (WIN) improves neurologic outcome and spinal neuron survival after ischemic spinal cord injury. A, The experimental design of the study. B, Differences of neurologic outcomes among the sham, control, and WIN groups 48 hours after reperfusion ( $\mathrm{n}=10$ per group). The neurologic outcome in the WIN group was better than that of control group. Data are presented as median \pm interquartile range. $\mathrm{C}$, The number of normal neurons in the anterior spinal cord of animals in the sham, control and WIN groups $(n=4$ per group). The data are presented as box and whiskers plot showing the median value, the 25 th and 75 th percentiles, and the range. Scale bars $=50 \mu \mathrm{m} . * * P<.01$ compared with sham group by Mann-Whitney tests. ${ }^{\# \#} P<.01$ compared with control group by Mann-Whitney tests. AM630, An antagonist of CB2 receptor; AM251, an antagonist of CB1 receptor.

\section{TUNEL Staining}

One day after surgery, apoptosis around the damaged region of the spinal cord was assessed with terminal deoxynucleotidyl transferase dUTP nick end labeling (TUNEL) staining (Roche Molecular Biochemicals, Indianapolis, Ind), as described in a previous study. ${ }^{17}$ The total number of stained cells in the spinal cord was counted and presented as cells per square millimeter ( $\mathrm{n}=4$ per group).

\section{Statistical Analysis}

All values were presented as mean \pm standard error of the mean except for the neurologic outcomes, which were expressed as median (interquartile range). Statistical analysis was performed with SPSS software version 13.0 (SPSS, Chicago, Ill). The neurologic results and the number of normal neurons in the anterior spinal cord were analyzed by the Mann-Whitney $U$ test. All remaining data were analyzed using one-way analysis of variance followed by the Dunnett test. A $P$ value less than .05 was considered statistically significant.

\section{RESULTS}

\section{WIN55212-2 Improves Neurologic Outcome and Spinal Neuron Survival After Ischemic SCI}

All animals survived before the neurologic behavior assessment at 48 hours after reperfusion. The MDI in the control group was greater than that of the sham group $(P<.05)$, while the WIN MDI was lower relative to the control group (Figure 1, B; Sham, 0 [0], vs Control, 5 [2], vs WIN, 2.5 [1.25]; $P<.05)$.

The number of normal motor neurons in the anterior spinal cord of control group (21.4 \pm 2.675 per section) was lower compared with the Sham group $(63.69 \pm 5.66$ per section; Figure $1, C ; P<.01 ; \mathrm{n}=4$ per group), while the WIN55212-2 group (43.1 \pm 3.69 per section) had more relative to the control group (Figure $1, C ; P<.01 ; \mathrm{n}=4$ per group).

\section{WIN55212-2 Blocks the Nuclear Translocation of GAPDH and Siah1}

We investigated whether the treatment of WIN55212-2 could affect the nuclear translocation of GAPDH and Siah1 in the spinal cord after ischemic SCI, and the ultimate complexing of the two. Our results showed that WIN552122 decreased the nuclear protein expression of GAPDH and Siah1 (Figure $2, A ; P<.01$ and $P<.01 ; \mathrm{n}=5$ per group). In addition, we also found that the decreases in the 


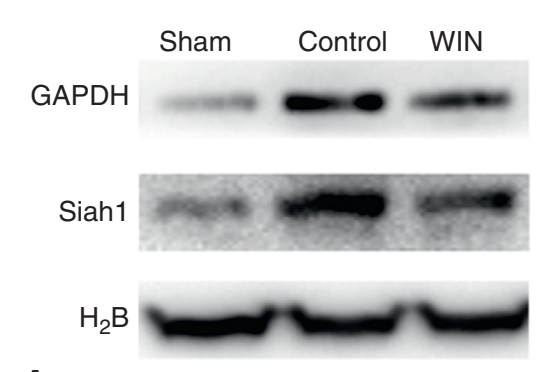

A

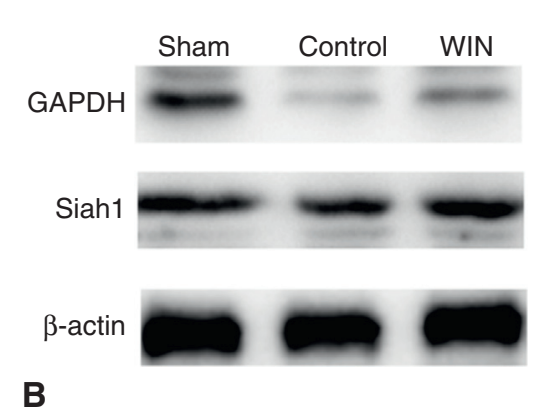

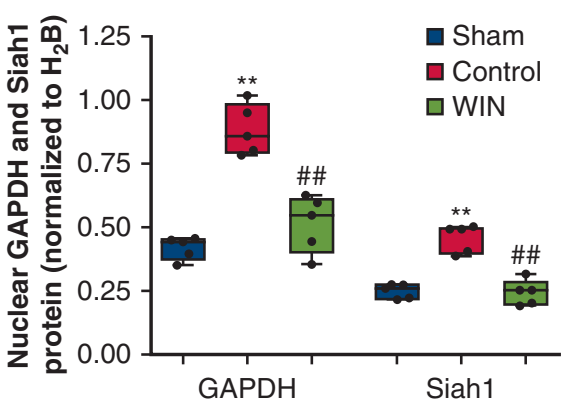

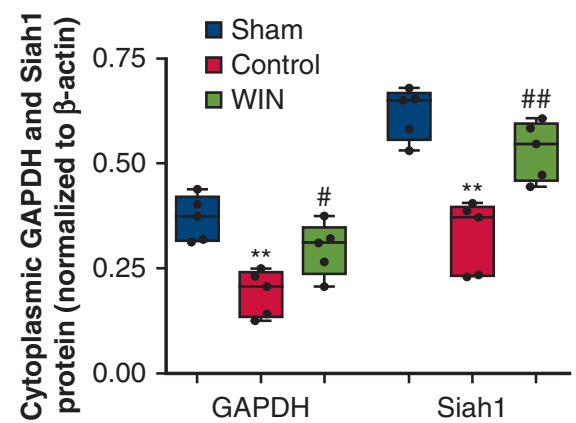

FIGURE 2. WIN55212-2 blocks the nuclear translocation of GAPDH and Siah1. A, A representative graph of Western blot showing the effects of WIN55212-2 on the nuclear protein expression of GAPDH and Siah1 in the spinal cord after ischemia ( $\mathrm{n}=5$ per group). The treatment of WIN552122 significantly reduced nuclear GAPDH and Siah1 protein expressions in the spinal cord after ischemia. The nuclear protein levels were normalized to histone $\mathrm{H}_{2} \mathrm{~B}$. B, A representative graph of Western blot showing the effects of WIN55212-2 on the cytoplasmic protein expression of GAPDH and Siah1 in the spinal cord after ischemia ( $n=5$ per group). The treatment of WIN55212-2 blocked the decrease of cytoplasmic GAPDH and Siah1 protein expressions in the spinal cord after ischemia. The cytoplasmic protein levels were normalized to $\beta$-actin. The data are presented as box and whiskers plot showing the median value, the 25 th and 75 th percentiles, and the range. ${ }^{* *} P<.01$ compared with sham group. ${ }^{\#} P<.05$ or ${ }^{\# \#} P<.01$ compared with control group. GAPDH, Glyceraldehyde-3-phosphate dehydrogenase; Siah1, E3 ubiquitin- protein ligase SIAH1; WIN, WIN55212-2.

cytoplasmic protein expressions of GAPDH and Siah1 were attenuated by WIN55212-2 (Figure $2, B ; P<.05$ and $P<.01 ; \mathrm{n}=5$ per group), as was the loss of complexing.

\section{WIN55212-2 Attenuates the Production of} Proinflammatory Cytokines and the Increase of iNOS Protein Expression in the Spinal Cord After Ischemia

We assessed the effects of WIN55212-2 on the production of proinflammatory cytokines in the spinal cord after ischemic SCI. Our data indicated that the production of IL- $1 \beta$ and TNF- $\alpha$ was reduced by WIN55212-2 treatment (Figure $3, A$, and $B ; P<.01$ and $P<.01 ; \mathrm{n}=5$ per group). The iNOS protein expression in the spinal cord was also assessed, and the results showed that its increase was alleviated following WIN55212-2 treatment (Figure 3, C; $P<.01 ; \mathrm{n}=5$ per group).

\section{The Improvement of Neurologic Outcome and the Blockade of GAPDH Nuclear Translocation Are Reversed by CB2 Receptor Antagonism}

It is still unknown which of the cannabinoid receptors is responsible for the beneficial effect of WIN55212-2 after ischemic SCI. Thus, AM251 (an antagonist of the CB1 receptor) or AM630 (an antagonist of the $\mathrm{CB} 2$ receptor) was coadministered with WIN55212-2. The MDI of the WIN+AM630 group was greater than that of the WIN55212-2 group (Figure $4, A ; P<.01 ; \mathrm{n}=10$ per group), while the differences between the WIN55212-2 and WIN+AM251 groups were not statistically significant: control group (5 [2]) versus WIN55212-2 group (2.5 [1.25]) versus WIN+AM630 group (5 [1.25]) versus WIN+AM251 group (2.5 [1.25]; Figure 4, $A ; P>.05$; $\mathrm{n}=10$ per group).

Next, we investigated whether the antagonism of CB1 or CB2 receptors could affect the inhibitory effect of WIN55212-2 on GAPDH/Siah1 signaling cascades. Our data indicated that the reduction of GAPDH nuclear translocation was nullified by the coadministration of AM630 (Figure $4, B ; P<.01 ; \mathrm{n}=5$ per group) and not AM251 (Figure $4, B ; P>.05 ; \mathrm{n}=5$ per group), suggesting that WIN55212-2 inhibits the GAPDH nuclear translocation through activation of $\mathrm{CB} 2$ receptors. Furthermore, we found that the immunoprecipitated GAPDH with anti-Siah1 antibodies was markedly reduced by the treatment of WIN55212-2 (Figure 4, $C ; P<.01 ; \mathrm{n}=4$ per group). Finally, the antagonism of CB2 receptors with AM630 blocked the reduction of the immunoprecipitated GAPDH induced by WIN55212-2 treatment (Figure 4, $C ; P<.01$; 


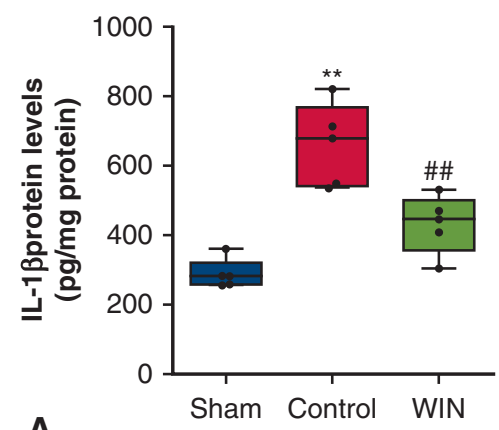

A
B
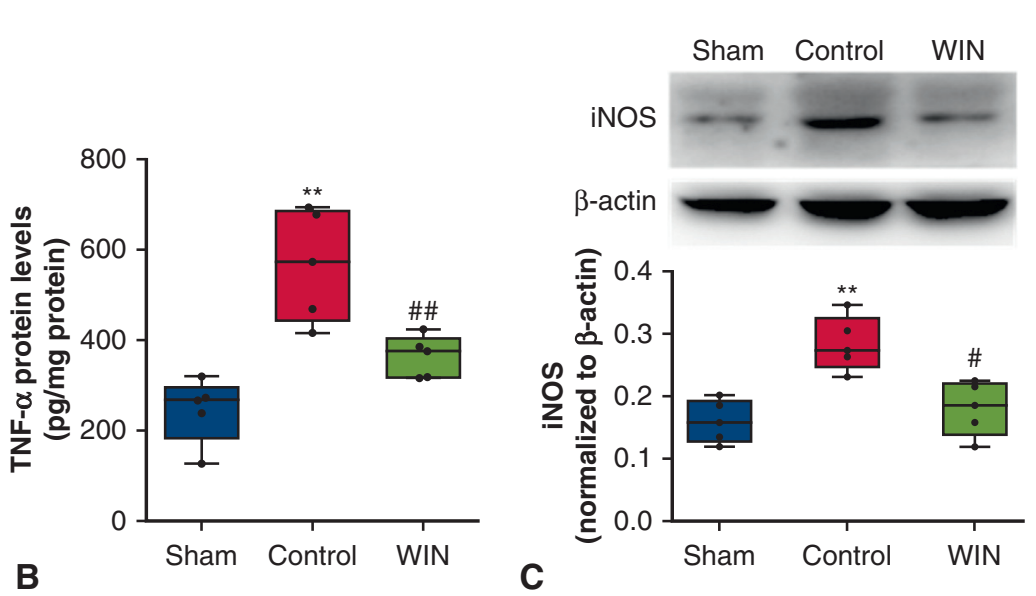

FIGURE 3. WIN55212-2 attenuates the production of proinflammatory cytokines and the increase of iNOS protein expression in the spinal cord after ischemia. The effects of WIN55212-2 on the protein levels of IL-1 $\beta$ (A) and TNF- $\alpha$ (B) were determined with Western blot ( $\mathrm{n}=5$ per group). C, A representative graph of Western blot showing the effects of WIN55212-2 on the protein levels of iNOS in the spinal cord after ischemia ( $\mathrm{n}=5$ per group). The data are presented as box and whiskers plot showing the median value, the 25 th and 75 th percentiles, and the range. $* * P<.01$ compared with sham group. ${ }^{\# \#} P<.01$ compared with control group. $I L-1 \beta$, Interleukin-1 beta; $i N O S$, inducible nitric oxide synthase; $T N F-\alpha$, tumor necrosis factor-alpha; WIN, WIN55212-2.

$\mathrm{n}=4$ per group), suggesting an essential role of CB2 receptors in the inhibition of GAPDH-Siah1 binding by WIN55212-2.

\section{WIN55212-2 Alleviates Apoptosis After Ischemic SCI in a CB2 Receptor-Dependent Manner}

Our results showed that WIN5512-2 significantly decreased the density of TUNEL-positive cells and the protein level of active caspase- 3 in the spinal cord after ischemia (Figure 5, $A$ and $B ; P<.01$ and $P<.01 ; \mathrm{n}=5$ per group). However, the antiapoptotic effect of WIN5512-2 treatment was reversed by the administration of AM630 (Figure 5, $A$ and $B ; P<.01$ and $P<.01 ; \mathrm{n}=5$ per group) and not AM251 (Figure 5, $A$ and $B ; P>.05$ and $P>.05 ; \mathrm{n}=5$ per group), indicating the CB2 receptor-dependent anti-apoptotic effect of WIN5512-2.

\section{WIN55212-2 Mitigates Inflammation After Ischemic SCI Through Activation of CB2 Receptor}

The antagonism of CB2 receptors with AM630 increased the protein levels of IL- $1 \beta$ and TNF- $\alpha$ in the WIN55212-2 animals (Figure 6, $A$ and $B ; P<.01$ and $P<.01 ; \mathrm{n}=5$ per group), whereas significant differences between the WIN55212-2 and WIN+AM251 groups were not found (Figure 6, $A$ and $B ; P>.01$ and $P>.01 ; \mathrm{n}=5$ per group). We next detected the activation of NF- $\kappa \mathrm{B}$ by quantifying the NF- $\kappa \mathrm{B}$ p65 subunit in nuclear and cytosolic fractions. Our data showed that the treatment of WIN55212-2 reduced the nuclear NF- $\kappa \mathrm{B}$ protein expression in the spinal cord after ischemic SCI along with the increase of cytoplasmic NF- $\kappa \mathrm{B}$ (Figure $6, C$ and $D$;
$P<.01$ and $P<.01 ; \mathrm{n}=5$ per group). In addition, it was also observed that the protein level of $\mathrm{I} \kappa \mathrm{B}-\alpha$ (inhibitor of NF- $\kappa \mathrm{B}$ ) in the cytosolic fraction was increased by the treatment of WIN55212-2 (Figure 6,D;P<.01 and $P<.01 ; \mathrm{n}=5$ per group), suggesting the prevention of $\mathrm{I} \kappa \mathrm{B}-\alpha$ degradation by WIN55212-2. Finally, the antagonism of $\mathrm{CB} 2$ receptors with AM630 blocked the inhibition of NF- $\kappa \mathrm{B}$ nuclear translocation and $\mathrm{I} \kappa \mathrm{B}-\alpha$ degradation caused by the WIN55212-2 treatment (Figure 6, $C$ and $D ; P<.05$ or $P<.01 ; \mathrm{n}=5$ per group). These results suggest that the anti-inflammatory effects of WIN55212-2 are also CB2 receptor-dependent.

\section{DISCUSSION}

GAPDH was once considered merely as a housekeeping protein in the cytosol. ${ }^{18}$ However, recent studies have revealed its essential roles in many cellular events, such as apoptosis ${ }^{19}$ and autophagy. ${ }^{20}$ Specifically, GAPDH would be initially S-nitrosylated by NO, and thereafter translocated into the nucleus to bind with Siah1, triggering apoptotic signaling cascades.$^{21}$ Importantly, our latest study showed that the overactivation of GAPDH/Siah1 signaling in the spinal cord is involved in traumatic SCI. In the present study, we aimed to investigate the potential role of GAPDH/ Siah1 signaling cascades in a model of ischemic SCI. We found that the nuclear translocation of GAPDH in the spinal cord was increased after ischemic insults. In addition, it was also observed that the elevation of GAPDH nuclear translocation was coupled with the increase in nuclear Siah1 expression and the formation of GAPDH/Siah1 complexes. Consistent with our results, a previous study of focal cerebral ischemia showed that GAPDH/Siah1 signaling can be 

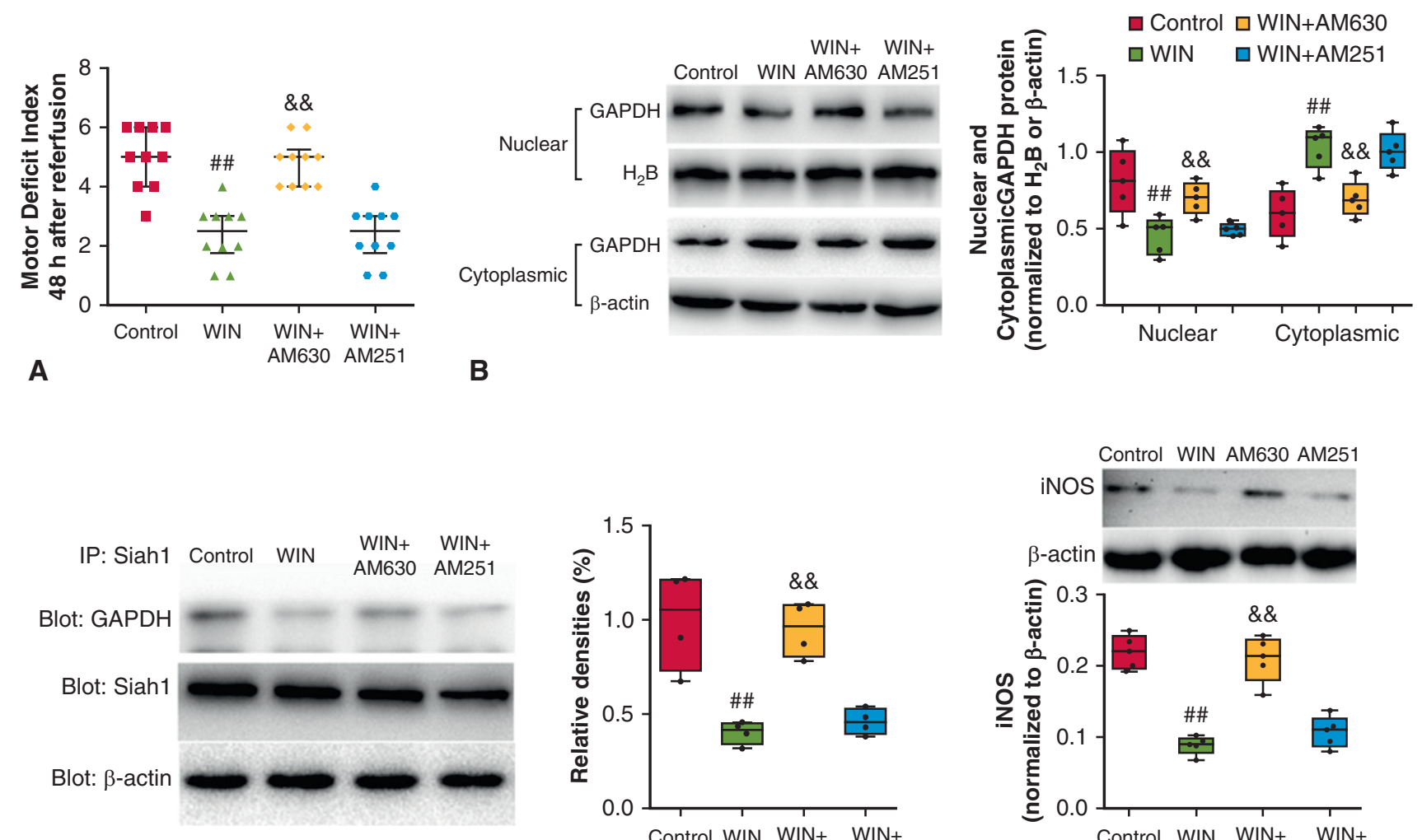

C

FIGURE 4. The improvement of neurologic outcome and the blockade of GAPDH nuclear translocation are reversed by the treatment of CB2 receptor antagonism. A, Differences of neurologic outcomes among the Control, WIN, WIN+AM251 or WIN+AM630 groups at 48 hours after reperfusion ( $n=10$ per group). Data are presented as median \pm interquartile range. B, A representative graph of Western blot showing the effects of combined treatments of AM251 or AM630 on the nuclear or cytoplasmic protein expression of GAPDH ( $\mathrm{n}=5$ per group). C, Immunoprecipitation of GAPDH with Siah1. The levels of immunoprecipitated GAPDH by anti-Siah1 antibodies were normalized to the corresponding input ( $\mathrm{n}=4$ per group). D, A representative graph of Western blot showing the effects of combined treatments of AM251 or AM630 on the protein expression of iNOS ( $\mathrm{n}=5$ per group). The data are presented as box and whiskers plot showing the median value, the 25 th and 75 th percentiles, and the range. ${ }^{\# \#} P<.01$ compared with sham group by Mann-Whitney tests. ${ }^{\& \&} P<.01$ compared with control group by Mann-Whitney tests. AM251, An antagonist of CB1 receptor; AM630, an antagonist of CB2 receptor; $G A P D H$, glyceraldehyde-3-phosphate dehydrogenase; $i N O S$, inducible nitric oxide synthase; IP, immunoprecipitation; Siah1, E3 ubiquitin- protein ligase SIAH1; WIN, WIN55212-2.

activated during the early stage of reperfusion and is correlated with the extent of apoptosis after ischemic injury. ${ }^{11}$ Together, these results indicate that GAPDH/Siah1 cascades could be initiated by ischemic insults and involved in the induction of apoptotic events.

Although the importance of the GAPDH/Siah1 signaling has been recognized, pharmacologic agents targeting these pathways are still lacking. The endogenous cannabinoid system has been identified with neuroprotective effects in multiple pathological conditions, and the upregulation of the endocannabinoid system might be a potential approach for inhibiting GAPDH/Siah1 cascades. WIN55212-2 is a synthetic cannabinoid agonist with demonstrated neuroprotective effects against global or focal cerebral ischemic injuries. ${ }^{22,23}$ Here, we showed that the systemic treatment with WIN55212-2 blocked GAPDH nuclear translocation and the formation of GAPDH/Siah1 complexes in the spinal cord after ischemia. This inhibitory action of WIN55212-2 on the GAPDH/Siah1 signaling cascade might be due to the suppression on NO production, as WIN55212-2 reduced the protein expression of iNOS in the spinal cord after IR injury.

Considering on the well-established role of GAPDH/ Siah1 cascade in apoptosis, ${ }^{19,20}$ the finding that apoptosis (TUNEL-positive cells and protein expression of active caspase-3) after IR injury was reduced by WIN55212-2 treatment was as expected. Importantly, the neuroprotective role of WIN55212-2 was supported by the improved neurologic scores after reperfusion in animals treated with WIN55212-2. The histologic results further confirmed that WIN55212-2 treatment promoted the survival of neurons in the spinal cord after reperfusion. These results are inconsistent with the previous studies in cerebral IR injury, 


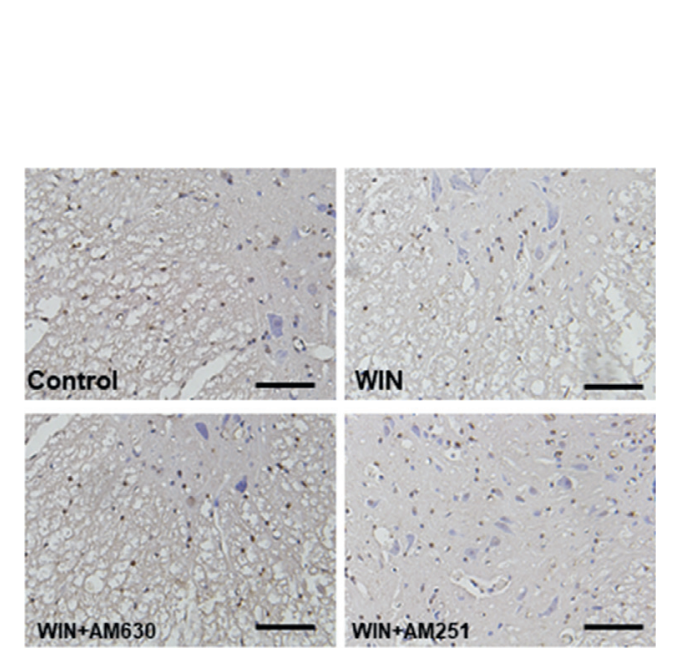

A

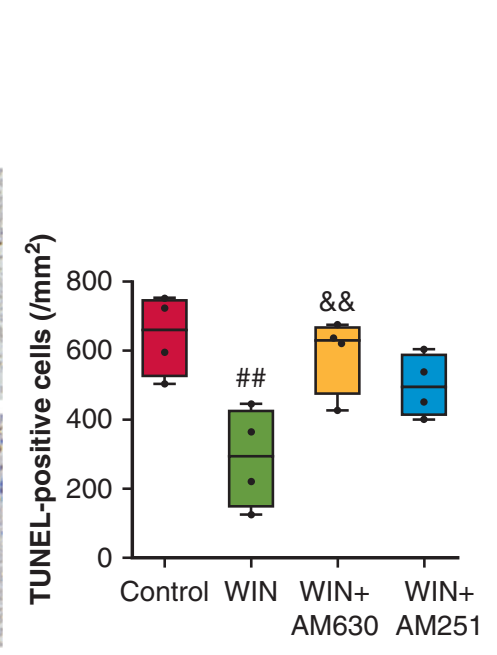

B

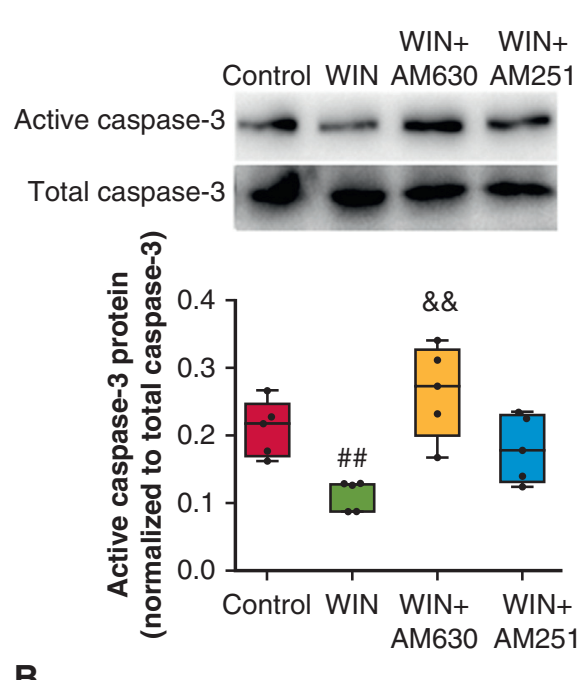

$\mathrm{WIN}+\mathrm{WIN}+$

Control WIN AM630 AM251

FIGURE 5. WIN55212-2 alleviates apoptosis after ischemic SCI in a CB2 receptor-dependent manner. A, A representative graph of Western blot showing the effects of WIN55212-2and the combined treatments of AM251 or AM630 on the density of TUNEL-positive cells in the spinal cord after ischemia $(\mathrm{n}=4)$. WIN55212-2markedly reduced the number of TUNEL-positive cells in the spinal cord after ischemic SCI, but this effect was reversed by the combined treatment of AM251. B, A representative graph of Western blot showing the effects of WIN55212-2 and the combined treatments of AM251 or AM630 on the protein expression of active (cleaved) caspase- 3 in the spinal cord after ischemia $(n=5)$. The data are presented as box and whiskers plot showing the median value, the 25 th and 75 th percentiles, and the range. ${ }^{\# \#} P<.01$ compared with control group. ${ }^{\& \&} P<.01$ compared with control group. Scale bars $=50 \mu \mathrm{m}$. WIN, WIN55212-2; AM251, an antagonist of CB1 receptor; AM630, an antagonist of CB2 receptor; TUNEL, terminal deoxynucleotidyl transferase dUTP nick end labeling.

which suggested that WIN55212-2 could reduce the infarct size and apoptosis and improve neurologic performance after ischemic injury. ${ }^{23}$

GAPDH/Siah1 signaling has also been implicated as a mediator of inflammatory and immune events in in vitro and in vivo studies. ${ }^{12,24,25}$ Our recent study has indicated that the activation of the GAPDH/Siah1 signaling pathway is associated with inflammation in the spinal cord after traumatic SCI. ${ }^{13}$ In the present study, our results indirectly support its regulatory role in inflammation by showing that the increase of proinflammatory cytokine production after ischemic SCI coincided with the activation of $\mathrm{GAPDH} / \mathrm{Siah} 1$. NF- $\kappa \mathrm{B}$ is a key mediator in inflammatory diseases $^{26}$ and IR injury. ${ }^{27}$ Previous studies have shown that WIN55212-2 significantly inhibits NF- $\kappa$ B activation in primary neurons ${ }^{28}$ and astrocytes. ${ }^{29}$ In accordance with these studies, we found that after reperfusion, WIN552122 blunted the nuclear translocation of NF- $\kappa \mathrm{B}$ and reduced cytosolic $\mathrm{I} \kappa \mathrm{B} \alpha$ degradation, indicative of reduced NF- $\kappa \mathrm{B}$ signaling activation. ${ }^{26}$

Another interesting question is whether the activation of $\mathrm{CB} 1$ or $\mathrm{CB} 2$ receptor is responsible for the neuroprotective effects of WIN55212-2 in ischemic SCI. It has been reported that the selective potency of WIN55212-2 to CB2 $(\mathrm{Ki}=0.3 \mathrm{nM})$ receptor is relatively higher than that of $\mathrm{CB} 1(\mathrm{Ki}=1.9 \mathrm{nM}) \cdot{ }^{30}$ Consistently, our data showed that the addition of the $\mathrm{CB} 2$ antagonist AM630, rather than the CB1 antagonist AM251, significantly negated the inhibitory effects of WIN55212-2 on apoptosis and inflammation. These results are indicative of CB2 receptor activation being the main protective mechanism used by WIN55212-2 in the ischemic SCI model. Although both CB1 and CB2 receptors are linked to improved spontaneous recovery after SCI in previous literature, ${ }^{31,32}$ our results demonstrated that only the CB2 receptor is involved in the improvement of neurologic performance induced by WIN55212-2. In addition to receptor selectivity, we argue that this phenomenon might be, at least partly, explained by their difference in cellular distribution and function in the spinal cord, as: (1) $\mathrm{CB} 1$ is constitutively located in neural cells such as neurons in the spinal cord, whereas CB2 is mainly present in immunomodulatory cells, immune infiltrates, and astrocytes; and (2) CB1 receptor in the CNS is mainly associated with the processing of pain-related information and the formation of neuropathic pain, ${ }^{33}$ although the $\mathrm{CB} 2$ receptor is responsible for the regulation of inflammatory response in the spinal cord $^{34}$ and could alter expression upon injury. ${ }^{32}$

In conclusion, our study demonstrates a neuroprotective effect of WIN55212-2 against spinal cord IR injury, manifested as improved neurologic scores and decreased apoptosis and inflammation. This neuroprotective effect of WIN55212-2 could be a result of the inhibition of 

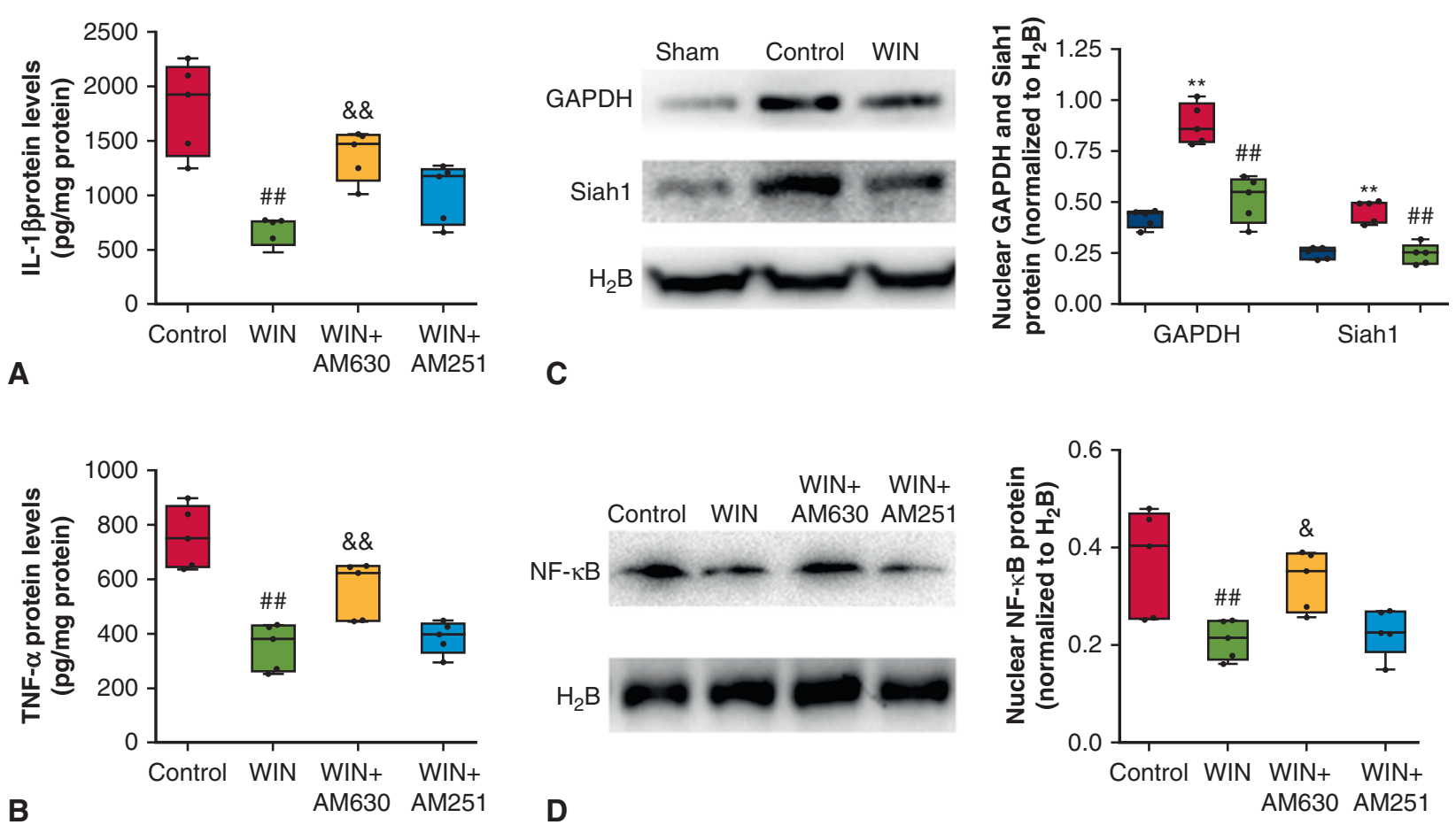

FIGURE 6. WIN55212-2 mitigates inflammation after ischemic SCI through activation of CB2 receptor. The effects of WIN55212-2and the combined treatments of AM251 or AM630 on IL-1 $\beta$ (A) and TNF- $\alpha$ (B) protein expression were determined with enzyme-linked immunosorbent assay. C, A representative graph of Western blot showing the effects of WIN55212-2 and the combined treatments of AM251 or AM630 on the NF- $\kappa$ B nuclear expression. The NF- $\kappa$ B nuclear protein level was normalized to histone $\mathrm{H}_{2} \mathrm{~B}$. D, A representative graph of Western blot showing the effects of WIN55212-2 and the combined treatments of AM251 or AM630 on the cytoplasmic protein expressions of NF- $\kappa \mathrm{B}$ and I $\kappa \mathrm{B}-\alpha$. The cytoplasmic protein levels were normalized to $\beta$-actin. The data are presented as box and whiskers plot showing the median value, the 25 th and 75 th percentiles, and the range (n $=5$ per group). ${ }^{* *} P<.01$ compared with sham group. ${ }^{\# \#} P<.01$ compared with control group. ${ }^{\&} P<.05$ or ${ }^{\& \&} P<.01$ compared with control group. IL-1 $\beta$, interleukin-1 beta; TNF- $\alpha$, tumor necrosis factor-alpha; WIN, WIN55212-2; AM251, an antagonist of CB1 receptor; AM630, an antagonist of CB2 receptor; I $K B$ - $\alpha$, nuclear factor of kappa light polypeptide gene enhancer in B-cells inhibitor, alpha; $N F-\kappa B$, nuclear factor kappa light-chain enhancer of activated B cells; $G A P D H$, glyceraldehyde-3-phosphate dehydrogenase; Siah1, E3 ubiquitin- protein ligase SIAH1.

GAPDH/Siah1 cascades through a CB2-dependent mechanism. These results reveal GAPDH/Siah1signalling cascades as a novel therapeutic target for ischemic SCI and identify WIN55212-2 or other synthetic cannabinoid agonist with the potential to manage ischemic SCI or other devastating ischemic conditions.

\section{Conflict of Interest Statement}

Authors have nothing to disclose with regard to commercial support.

\section{References}

1. Amantea D, Spagnuolo P, Bari M, Fezza F, Mazzei C, Tassorelli C, et al. Modulation of the endocannabinoid system by focal brain ischemia in the rat is involved in neuroprotection afforded by 17 $\beta$-estradiol. FEBS J. 2007;274: 4464-775.

2. Abood ME, Rizvi G, Sallapudi N, McAllister SD. Activation of the CB 1 cannabinoid receptor protects cultured mouse spinal neurons against excitotoxicity. Neurosci Lett. 2001;309:197-201.

3. Su B, Dong H, Ma R, Zhang X, Ding Q, Xiong L. Cannabinoid 1 receptor mediation of spinal cord ischemic tolerance induced by limb remote ischemia preconditioning in rats. J Thorac Cardiovasc Surg. 2009;138:1409-16.
4. Arévalo-Martín Á, García-Ovejero D, Gomez O, Rubio-Araiz A, NavarroGalve B, Guaza C, et al. CB2 cannabinoid receptors as an emerging target for demyelinating diseases: from neuroimmune interactions to cell replacement strategies. Br J Pharmacol. 2008;153:216-25.

5. Di Marzo V. Targeting the endocannabinoid system: to enhance or reduce? Nat Rev Drug Discov. 2008;7:438-55.

6. Fernández-López D, Pradillo JM, García-Yébenes I, Martínez-Orgado JA, Moro MA, Lizasoain I. The cannabinoid WIN55212-2 promotes neural repair after neonatal hypoxia-ischemia. Stroke. 2010;41:2956-64.

7. Hama A, Sagen J. Antinociceptive effect of cannabinoid agonist WIN 55,212-2 in rats with a spinal cord injury. Exp Neurol. 2007;204:454-7.

8. Desroches J, Charron S, Bouchard J-F, Beaulieu P. Endocannabinoids decrease neuropathic pain-related behavior in mice through the activation of one or both peripheral CB 1 and CB 2 receptors. Neuropharmacology. 2014;77:441-52.

9. Hara MR, Snyder SH. Nitric oxide-GAPDH-Siah: a novel cell death cascade. Cell Mol Neurobiol. 2006;26:525-36.

10. Hara MR, Cascio MB, Sawa A. GAPDH as a sensor of NO stress. Biochim Biophys Acta. 2006;1762:502-9.

11. Li C, Feng J-J, Wu Y-P, Zhang G-Y. Cerebral ischemia-reperfusion induces GAPDH S-nitrosylation and nuclear translocation. Biochemistry (Mosc). 2012; 77:671-8.

12. Takaoka Y, Goto S, Nakano T, Tseng HP, Yang SM, Kawamoto S, et al. Glyceraldehyde-3-phosphate dehydrogenase (GAPDH) prevents lipopolysaccharide (LPS)-induced, sepsis-related severe acute lung injury in mice. Sci Rep. 2014; 4:5204. 
13. Huo J, Zhu X-L, Ma R, Dong H-L, Su B-X. GAPDH/Siah1 cascade is involved in traumatic spinal cord injury and could be attenuated by sivelestat sodium. Neuroscience. 2016;330:171-80.

14. Cabral GA, Harmon KN, Carlisle SJ. Cannabinoid-Mediated Inhibition of Inducible Nitric Oxide Production by Rat Microglial Cells: Evidence for CB1 Receptor Participation. Neuroimmune Circuits, Drugs of Abuse, and Infectious Diseases. Boston: Springer; 2002:207-14.

15. Molina-Holgado F, Molina-Holgado E, Guaza C, Rothwell NJ. Role of CB1 and CB2 receptors in the inhibitory effects of cannabinoids on lipopolysaccharide-induced nitric oxide release in astrocyte cultures. J Neurosci Res. 2002;67:829-36.

16. González C, Herradón E, Abalo R, Vera G, Pérez-Nievas BG, Leza JC, et al. Cannabinoid/agonist WIN 55,212-2 reduces cardiac ischaemia-reperfusion injury in Zucker diabetic fatty rats: role of CB2 receptors and iNOS/eNOS. Diabetes Metab Res Rev. 2011;27:331-40.

17. Sasaki M, Hains BC, Lankford KL, Waxman SG, Kocsis JD. Protection of corticospinal tract neurons after dorsal spinal cord transection and engraftment of olfactory ensheathing cells. Glia. 2006;53:352-9.

18. Nicholls C, Li H, Liu JP. GAPDH: a common enzyme with uncommon functions. Clin Exp Pharmacol Physiol. 2012:39:674-9.

19. Hara MR, Agrawal N, Kim SF, Cascio MB, Fujimuro M, Ozeki Y, et al. S-nitrosylated GAPDH initiates apoptotic cell death by nuclear translocation following Siah1 binding. Nat Cell Biol. 2005;7:665-74.

20. Colell A, Ricci J-E, Tait S, Milasta S, Maurer U, Bouchier-Hayes L, et al. GAPDH and autophagy preserve survival after apoptotic cytochrome c release in the absence of caspase activation. Cell. 2007;129:983-97.

21. Sen N, Hara MR, Kornberg MD, Cascio MB, Bae BI, Shahani N, et al. Nitric oxide-induced nuclear GAPDH activates p300/CBP and mediates apoptosis. Nat Cell Biol. 2008;10:866-73.

22. Nagayama T, Sinor AD, Simon RP, Chen J, Graham SH, Jin K, et al. Cannabinoids and neuroprotection in global and focal cerebral ischemia and in neuronal cultures. J Neurosci. 1999;19:2987-95.

23. Hu B, Wang Q, Chen Y, Du J, Zhu X, Lu Y, et al. Neuroprotective effect of WIN 55,212-2 pretreatment against focal cerebral ischemia through activation of extracellular signal-regulated kinases in rats. Eur J Pharmacol. 2010;645:102-7.

24. Raje CI, Kumar S, Harle A, Nanda JS, Raje M. The macrophage cell surface glyceraldehyde-3-phosphate dehydrogenase is a novel transferrin receptor. $J$ Biol Chem. 2007;282:3252-61.
25. Zhou Y, Yi X, Jha'Nae BS, Bonafe N, Gilmore-Hebert M, McAlpine J, et al. The multifunctional protein glyceraldehyde-3-phosphate dehydrogenase is both regulated and controls colony-stimulating factor-1 messenger RNA stability in ovarian cancer. Mol Cancer Res. 2008;6:1375-84.

26. Tak PP, Firestein GS. NF- $\kappa$ B: a key role in inflammatory diseases. J Clin Invest. 2001; 107:7-11.

27. Ridder D, Schwaninger M. NF- $\kappa$ B signaling in cerebral ischemia. Neuroscience 2009;158:995-1006

28. Jüttler E, Potrovita I, Tarabin V, Prinz S, Dong-Si T, Fink G, et al. The cannabinoid dexanabinol is an inhibitor of the nuclear factor-kappa B (NF- $\kappa \mathrm{B})$. Neuropharmacology. 2004;47:580-92.

29. Aguirre-Rueda D, Guerra-Ojeda S, Aldasoro M, Iradi A, Obrador E, Mauricio MD, et al. WIN 55,212-2, Agonist of cannabinoid receptors, prevents amyloid $\beta$ 1-42 effects on astrocytes in primary culture. PloS One. 2015;10: $\mathrm{e} 0122843$.

30. Showalter VM, Compton DR, Martin BR, Abood ME. Evaluation of binding in a transfected cell line expressing a peripheral cannabinoid receptor (CB2): identification of cannabinoid receptor subtype selective ligands. J Pharmacol Exp Ther. 1996;278:989-99.

31. Arevalo-Martin A, Garcia-Ovejero D, Sierra-Palomares Y, PaniaguaTorija B, Gonzalez-Gil I, Ortega-Gutierrez S, et al. Early endogenous activation of $\mathrm{CB} 1$ and $\mathrm{CB} 2$ receptors after spinal cord injury is a protective response involved in spontaneous recovery. PloS One. 2012;7: e49057.

32. Garcia-Ovejero D, Arevalo-Martin A, Petrosino S, Docagne F, Hagen C, Bisogno T, et al. The endocannabinoid system is modulated in response to spinal cord injury in rats. Neurobiol Dis. 2009;33:57-71.

33. Talwar R, Kumar Potluri V. Cannabinoid 1 (CB1) receptor-pharmacology, role in pain and recent developments in emerging CB1 agonists. CNS Neurol Disord Drug Targets. 2011;10:536-44.

34. Adhikary S, Li H, Heller J, Skarica M, Zhang M, Ganea D, et al. Modulation of inflammatory responses by a cannabinoid-2-selective agonist after spinal cord injury. J Neurotrauma. 2011;28:2417-27.

Key Words: WIN55212-2, ischemic spinal cord injury, GAPDH, cannabinoid receptors 

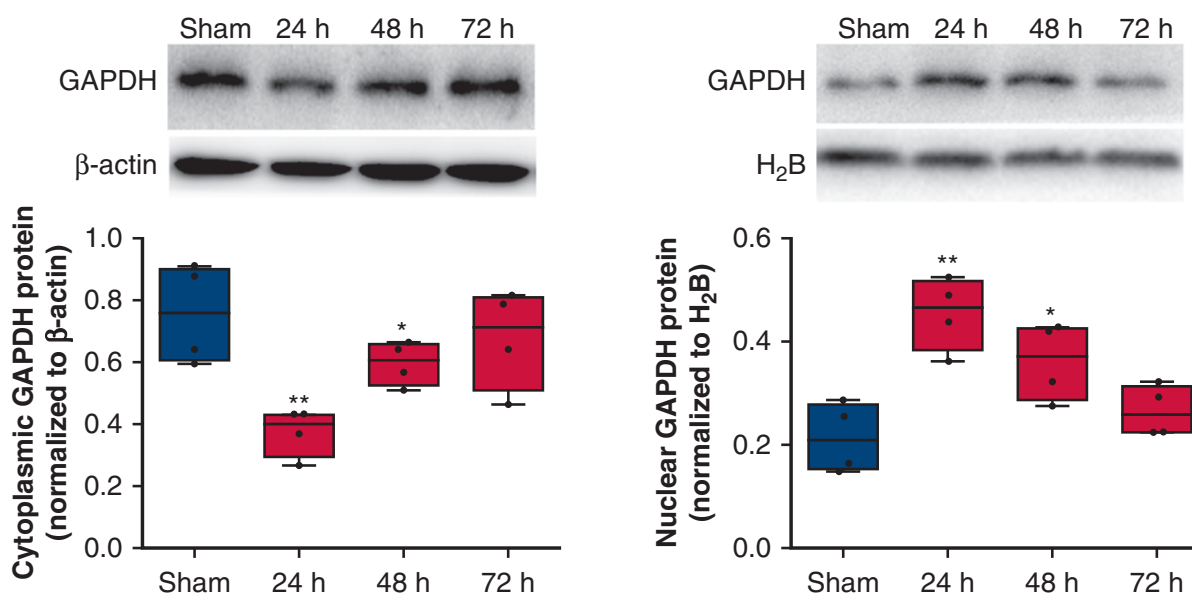

A

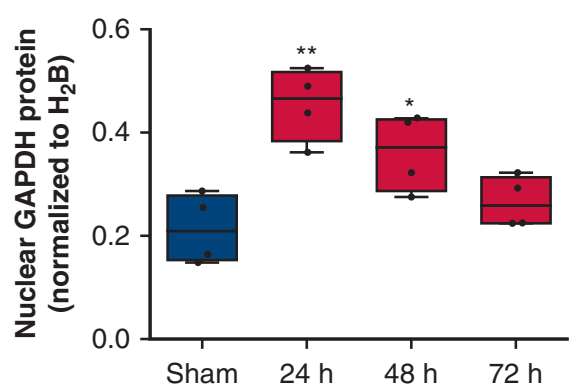

B

FIGURE E1. Time pattern of the nuclear and cytoplasmic glyceraldehyde 3-phosphate dehydrogenase (GAPDH) protein expression after ischemic spinal cord injury (SCI). A, The time pattern of the nuclear protein level of GAPDH after ischemic SCI was detected by Western blot analysis ( $\mathrm{n}=4$ per group). The nuclear protein expressions of GAPDH were normalized to histone $\mathrm{H}_{2} \mathrm{~B}$. Nuclear GAPDH expressions increased after reperfusion, peaked at 24 hours, and then began to decrease. B, The time pattern of cytoplasmic GAPDH after ischemic SCI ( $\mathrm{n}=4$ per group). The cytoplasmic protein expression of GAPDH were normalized to $\beta$-actin. Data are presented as mean \pm standard error of the mean. $* P<0.05$ or $* * P<0.01$ compared with sham. 